\section{PRÁCTICAS EN LUGARES DE TRABAJO EN LOS PROGRAMAS \\ DE EMPLEO JUVENIL: EXPERIENCIAS, CAMBIOS Y CONTINUIDADES EN SU IMPLEMENTACIÓN}

\author{
WORK PLACEMENTS IN YOUTH EMPLOYMENT PROGRAMS: \\ EXPERIENCES, CHANGE AND CONTINUITY IN \\ THEIR IMPLEMENTATION
}

\author{
José Antonio Pozzer
}

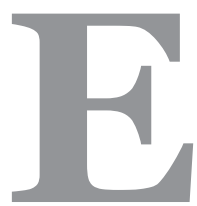

n el marco de la creciente implementación de políticas y programas dirigidos a acompañar las inserciones juveniles al mercado de trabajo, se proponen algunas experiencias con componentes que buscan la articulación del Estado y las empresas. Este artículo revisa los supuestos y estrategias desde los cuales se gestionan e implementan -por parte de ambos actores- las prácticas en espacios de trabajo, a partir de la revisión de tres (3) programas específicos que plantearon el componente en Argentina: Plan Joven (1993-200o); Programa Jóvenes con Futuro (20072010) y Programa Jóvenes con Más y Mejor Trabajo -línea "Entrenamiento"(2008-continua). El análisis se basa en la revisión de información documental y entrevistas a actores vinculados a su implementación. Se pregunta y muestra cómo en el transcurso de más de veinticinco (25) años de ejecución de estas líneas con componentes de prácticas se han producido ciertos cambios en la concepción y abordaje de la problemática de empleo juvenil, como así también en las estrategias seguidas para su gestión, pese a lo cual los resultados alcanzados y la intervención concreta han mostrado resultado similares.

Palabras clave: políticas públicas de empleo, jóvenes, formación en el trabajo.

\section{ABSTRACT}

Taking into account the framework of the growing implementation of policies and programs aimed at accompanying youth insertions in the labor market, some experiences are proposed with components that seek
José Antonio Pozzer japozzer@yahoo.com.ar

CONICET

Facultad de Humanidades y Facultad de Ciencias Económicas

Universidad Nacional del Nordeste ARGENTINA

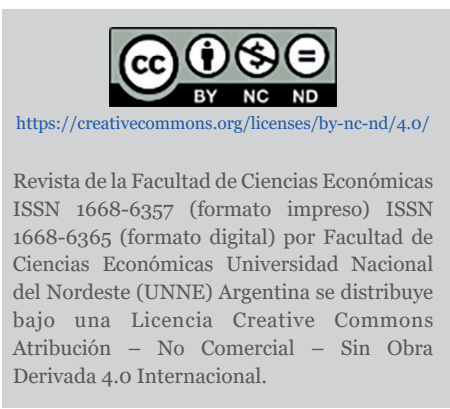


the articulation of the State and companies. With this in mind, the premises and plans are based on the revision of three (3) specific programs that have suggested this action strategy in Argentina: "Plan Joven" (1993-2000); "Jóvenes con Futuro" (2007-2010) and “Jóvenes con Más y Mejor Trabajo”-componente "Entrenamiento"- (2008-continue). The analysis is based on the revision of documentary information and interviews with the actors involved in its implementation. As a result of this, it can be shown that during more than twenty-five (25) years of the application of these practical programs and components there have been a considerable development not only in the conception and approach of youth employment issue, but also in the strategies used for its management. Notwithstanding, the results accomplished and the particular intervention on the problem have shown similar results.

Keywords: public employment policies, young, job training .

\section{PRESENTACIÓN}

En este artículo abordaremos algunos supuestos y estrategias de programas dirigidos a intermediar en los procesos de inserción laboral de jóvenes que proponen el desarrollo de prácticas en espacios de trabajo y que implican la articulación de actores estatales y empresariales en su gestión e implementación.

Desde hace aproximadamente tres décadas, la inserción laboral de los jóvenes constituye un tema central en las discusiones sobre la cuestión social, porque con la crisis de los estados de bienestar y del pleno empleo, lo que se pone en juego son los mecanismos sistémicos de integración social (Jacinto, 2010). La inserción laboral de jóvenes, que tradicionalmente había sido representada por una secuencia de pasos institucionalizados que iban de la educación al trabajo, muestra nuevos recorridos más difusos e inestables. Particularmente para los jóvenes de menores recursos se presenta -además- el riesgo de encontrarse ligados a la precariedad e informalidad en las ocupaciones.

En este marco, durante la década de los `9o “aparecen” en la escena de las políticas públicas en América Latina y en Argentina, los primeros programas enfocados en la inserción laboral de los jóvenes con baja escolaridad formal. Orientados por diagnósticos inespecíficos sobre los problemas del mercado de trabajo, se concentraron solo en los "déficits" de formación de los propios jóvenes -pocos años de escolaridad formal y la ausencia de capacitación necesaria demandada por el mercado-, a la vez que sostenían una visión crítica sobre las regulaciones protectoras del empleo, que resultaban poco flexibles y no favorables a la demanda de mano de obra joven (Moura Castro, 2002). Las acciones implementadas tuvieron un carácter com- 
pensatorio, y se vincularon a actividades de capacitación laboral inicial, pasantías y apoyo a microemprendimientos.

Iniciados los años 2000, se produjeron algunas reformulaciones de las iniciativas de este tipo, respondiendo a la noción de empleabilidad como eje conceptual de acción sobre la problemática del empleo juvenil. Así, las políticas de su tipo pasaron de las intervenciones indemnizatorias por desempleo a la movilización de la oferta de trabajo o activación. Desde esta perspectiva, una renovada idea de empleabilidad propone una articulación entre elementos estructurales del mercado de trabajo y subjetivos de las personas, desde donde se entiende que el problema del empleo juvenil debe explicarse, por un lado, por el desarreglo de expectativas de los jóvenes "inempleables" y las demandas laborales reales y, por el otro, por un déficit de competencias o, en otras palabras, de capital humano y de educación (Salvia, 2013).

De este modo, el cambio de siglo -y de condiciones del mercado de trabajo- produjo un viraje hacia la perspectiva de orientación e inducción al mundo del trabajo (Pérez, 2013; Jacinto, 2010/2008). En esta construcción del problema, la política activa ya no identificaría una falta de competencias técnicas específicas en los jóvenes vulnerables para ocupar empleos calificados, sino más bien un déficit de competencias básicas y transversales, para la búsqueda, la consecución y el mantenimiento de empleos.

Así vemos que en los últimos veinticinco años, se han implementado de manera casi sucesiva algunos programas de gran envergadura -considerando la cobertura territorial y cantidad de personas-, sin embargo, tal como señala OIT (2016), en general poco se conoce respecto de la amplitud y el alcance de las políticas activas del mercado de trabajo. En particular, nos interesan aquellas que propusieron dentro de sus prestaciones el desarrollo de prácticas en espacios de trabajo y que involucraron la participación del actor empresarial/empleadores: Plan Joven (1993-2000); Jóvenes con Futuro (2007-2010); Jóvenes con Más y Mejor Trabajo (2008-continua). A partir de los diferentes contextos y marcos que orientaron las acciones, realizamos una revisión de las propuestas programáticas desarrolladas, su ejecución y algunos resultados/impactos sobre la problemática de la inserción.

El análisis realizado proviene de diferentes fuentes y alcances, para el Plan Joven y el Jóvenes con Futuro, de la revisión documental de informes de gestión y estudios de impacto y resultados (su gestión era centralizada, por lo cual solo muestran datos a nivel nacional, no desagregados por región/localidad); y para el Programa Jóvenes con Más y Mejor Trabajo, de la revisión de documentos (decretos/resoluciones; manuales de implementación e informes de gestión) y entrevistas a actores vinculados a su ejecución en Chaco y Corrientes (coordinadores de oficinas de empleo; vinculadores de empresas; referentes de empresas; jóvenes participantes). En este último caso, el programa apunta a la descentralización de su gestión en los contextos locales, tomando relevancia este nivel de análisis. 


\subsection{Políticas activas de mercado de trabajo y empleo}

Como mencionáramos anteriormente, la OIT (2016) señalaba que en América Latina se conoce poco respecto de la amplitud y el alcance de las políticas activas del mercado de trabajo (en adelante PAMT) puestas en marcha en la región y, en particular, sobre algunos programas como los servicios del mercado de trabajo y las subvenciones al empleo.

La mirada desde el Estado sobre los jóvenes como sujeto de políticas viene instalándose en la agenda pública desde hace casi tres décadas (Jacinto, 2015). En este tiempo han sido variadas las intervenciones en materia de inclusión socio-laboral, observándose tanto medidas pasivas (centradas en la oferta, a través de asistencia dineraria al desempleo sin contraprestación) como activas (intervienen sobre la oferta y la demanda a la vez, implican subsidios a cambio de la movilización a través de alguna actividad), que buscaron tener alguna repercusión tanto en la formación profesional como en la demanda de empleo hacia los jóvenes y, en menor medida, corregir asimetrías de oportunidades y facilitar canales de acceso a una mejor inserción laboral (Neffa, 2012; Salvia, 2008; Lasida, 2004; Salvia y Tuñón, 2003).

Una revisión conceptual de estas políticas muestra sus múltiples objetivos y ámbitos de intervención. Tradicionalmente, se orientaban a mantener a las personas activas en el mercado de trabajo y mejorar la reinserción laboral. Sin embargo, con el tiempo han tomado una orientación que sobrepasa el objetivo de reducir el desempleo. En la práctica, pueden incluir el apoyo a los trabajadores en el marco de una transición de un sector a otro; distintos programas destinados a mantener los ingresos de individuos durante periodos de recesión o completar los salarios de grupos vulnerables para así reducir la pobreza o la desigualdad (OIT, 2016).

En este marco, se ha consolidado el "paradigma de activación" de las políticas sociales como la forma de trabajar sobre el problema del desempleo, a través de una diversidad de dispositivos. La lógica que subyace en ellos es que los desocupados no "carecen" de todo, sino que poseen ciertos atributos y condiciones que podrían movilizar para superar su condición. De este modo mediante las políticas activas de empleo se busca proveerlos de una cartera de activos que les permita mejorar su capital humano y social (Pérez y Brown, 2015).

A continuación presentaremos una revisión de los modos en que fue desarrollándose en diferentes momentos en nuestro país.

\section{CONFIgURACIÓN DE LA POLÍtica DE EMPLEO PARA JÓVENES EN ARGENTINA: ¿30 AÑOS DE CONTINUIDADES?}

Con la sanción de la Ley Nacional de Empleo (LNE) $\mathrm{N}^{\circ} 24.013$ en diciembre de $1991^{1}$, se introdujeron una serie de medidas pasivas y activas para hacer frente al avance del desempleo y la degradación de las condiciones laborales de los trabajadores. La ley tenía como objetivos

\footnotetext{
${ }^{1}$ Ley "fundante" en materia de intervenciones de política laboral, como la creación de "grupos especiales de trabajadores" en referencia a los desocupados.
} 
principales, regular los efectos de los procesos de "reconversión productiva" y de reforma estructural sobre el empleo. Bajo la órbita de aplicación del Ministerio de Trabajo y Seguridad Social (MTESS), se planteó promover el desarrollo de políticas tendientes a incrementar la producción y la productividad, fomentar las oportunidades de empleo para los grupos que enfrentaban mayores dificultades de inserción laboral e incorporar la formación profesional como componente básico de las políticas y programas de empleo.

De acuerdo con las orientaciones de la LNE, todos los programas que se crearan tenían como eje de fomento del empleo y debían centrarse en: “a) actualización y reconversión profesional hacia ocupaciones de expansión más dinámica; b) orientación y formación profesional; c) asistencia en caso de movilidad geográfica; d) asistencia técnica y financiera para iniciar pequeñas empresas, principalmente en forma asociada" (Art.82, Cap.3, Ley 24.013).

Dentro del marco normativo se pudieron identificar al menos dos modalidades promovidas que implican especialmente a los jóvenes: los contratos de práctica laboral y los contratos de trabajo-formación. También se introdujeron programas de duración determinada para jóvenes sin empleo, bajo la premisa: "Estos programas atenderán a las personas desocupadas entre 14 y 24 años". Así, se previeron acciones a través de "programas activos" con orientación al empleo de los jóvenes, práctica laboral para jóvenes con y sin formación previa, fomento de trabajo intensivo en mano de obra desde gobiernos municipales, organizaciones no gubernamentales y micro emprendimientos privados, y formación profesional.

Los marcos en que se orientaron las acciones estuvieron permeadas por la idea de "empleabilidad"; el desarrollo de "competencias laborales" en los programas con componentes de capacitación laboral; medidas de flexibilización en la contratación de la mano de obra, baja de los costos laborales, disminución de impuestos y contribuciones laborales para las empresas a través de la creación de diversos formatos contractuales (Bowman, 2015).

Particularmente se instrumentó una oferta basada en pasantías y en formación focalizada -por ejemplo el Programa Joven-, a través de cursos de corta duración y de capacitación, que debía responder exclusivamente a las demandas del sector productivo, ámbito desde donde empresarios y capacitadores definían los perfiles ocupacionales requeridos y el diseño de las propuestas pedagógicas. Varias autoras (Jacinto 2008/1997; Gallart, 2001; Riquelme y Herger, 2001) coinciden en el análisis de estas acciones de capacitación laboral, al señalarlos como fragmentados, pragmáticos, con escasas articulaciones con la formación profesional regular, con la educación básica obligatoria y con las políticas integrales de desarrollo.

Los programas se concentraron en gran medida en la capacitación laboral orientada al empleo formal o a la inserción en el autoempleo o en emprendimientos productivos (Jacinto, 2004). Se fundamentaron en torno al presupuesto de que la baja "empleabilidad" de los jóvenes tenía origen/causa en su escaso capital humano (Moura Castro, 2002), y que ante los cambios en las de- 
mandas de calificaciones, su falta o escasez "explicaba" el motivo de las problemáticas de inserción. Sin embargo, tal como lo señala Jacinto (2008) esta argumentación planteaba una visión parcial en la que se omitían las desigualdades estructurales y la segmentación del mercado de trabajo. ${ }^{2}$

En este marco, la falta de integralidad y la parcialidad en la que se basó la política de empleo se han presentado como una "deuda" en relación con los programas desarrollados en la década del 90. Fue visualizada asimismo como la causa de muchas falencias operativas, de las dificultades de sostenimiento en el tiempo y de la armonización local de políticas emanadas de diferentes áreas del gobierno central, de los inconvenientes para sistematizar y ordenar acciones, entre otras cuestiones (Bowman, 2015; Jacinto, 2010; Devia, 2003).

Los años 2000, posteriores a la crisis social, económica y política de Argentina ${ }^{3}$ ¿̇marcaron un punto de cambio en la política de empleo para jóvenes? La gestión llevada a cabo por el MTESS se realizó desde un marco basado en el desarrollo territorial. Desde este enfoque la política asumió la función de fortalecer la construcción de estrategias colaborativas y de diseñar herramientas que se adapten a las dinámicas de los espacios locales, promoviendo canales de coordinación entre los diferentes niveles de la administración pública y articulación con distintos actores (privados, sociales, académicos, etc.). La cuestión de la territorialidad se proponía como camino para reconstruir las bases sociales de una "nueva política" (Madoery, 2011), como una forma de superar la fragmentación y la superposición de la política de empleo, y los "efectos modestos" que habían mostrado las intervenciones precedentes.

La estrategia del MTESS se asentó en tres pilares: los servicios públicos de empleo, la formación profesional y el desarrollo económico local. Con el apoyo de la OIT y de otros organismos internacionales de crédito como el BID, desde la cartera laboral se impulsaron en el periodo 2003-2008 un conjunto de políticas de empleo, entre las que se destacan: el "Plan Integral de Promoción del Empleo Más y Mejor Trabajo" (2003), el fortalecimiento de la "Red de Oficinas Municipales de Empleo" (2005), el "Programa de Formación para el Trabajo” (2006), y el "Programa Jóvenes con Más y Mejor Trabajo (2008) focalizado en jóvenes con baja escolaridad.

En términos generales, todas ellas proponían un cambio de modelo basado en la activación (Jacinto, 2010). Esto implicaba desarrollar medidas que no se limitaban a la transferencia de un subsidio, sino que constituyan incentivos a la participación activa de los individuos en su acercamiento al trabajo. Se planteaban desde una mirada de involucramiento en la construcción de la trayectoria laboral, donde los jóvenes desocupados se movilicen para mejorar y/o modificar su situación (Pérez y Brown, 2015; Partenio, 2015), supuestamente asegurada por la existencia de una contraprestación.

\footnotetext{
${ }^{2}$ La idea de la "baja empleabilidad" de los jóvenes se planteaba en un momento en que el desempleo aumentaba, pero se atribuía al propio sujeto esas dificultades. En 1995 la tasa de desempleo de la PEA en el total de aglomerados relevados por la EPH-INDEC era del 18,4\%, mientras que para la población joven (menores de 25 años) ascendía al 34,4\%.

${ }^{3}$ Desencadenada en diciembre de 2001, como consecuencia de una creciente recesión económica iniciada luego de 1998 , que tuvo un alto impacto social, alcanzado un 50\% de pobreza y alrededor de $20 \%$ de desocupación. La crisis se amplió a cuestiones de representatividad política e institucional, potenciada por una revuelta popular generalizada bajo el lema "iQue se vayan todos!", que causó la renuncia del entonces presidente Fernando de la Rúa, dando lugar a un período de inestabilidad institucional durante el cual cinco funcionarios ejercieron la Presidencia de la Nación en el transcurso de un mes.
} 
En este marco, empiezan a desarrollarse propuestas que ponen en diálogo elementos estructurales e individuales del mercado de trabajo. Se entiende que el problema del empleo juvenil debe explicarse, por un lado, por el desarreglo de expectativas de los jóvenes "inempleables" y las demandas laborales reales (Salvia, 2008) y, por el otro, por un déficit de competencias (Salvia, 2013). Al respecto, Jacinto $(2008,2010)$ señala que se produjo un viraje hacia la perspectiva de orientación e inducción al mundo del trabajo.

Varios estudios coinciden en mostrar que los programas se centraron en la regulación y formación de aspectos subjetivos, tales como la formación actitudinal, de mejoramiento de la autoestima, de incentivo a la motivación para el trabajo (Pérez, 2013) y de habilidades comunicacionales e interaccionales para el mundo laboral, a partir de intervenciones personalizadas y de procesos de acompañamiento permanente (Jacinto y Millenaar, 2009).

De este modo, la "formación para la empleabilidad" 4 desde la perspectiva del Trabajo Decente (fundamento de organismos internacionales que financiaron programas), implica apoyar a las personas para que "valoren sus habilidades" y sus saberes así como las demandas y conocimientos requeridos en el mundo del trabajo (información, orientación e intermediación sobre la oferta de educación y del mercado laboral). Implica asimismo, la ayuda para desplegar la diversidad de alternativas, sus exigencias y sus posibilidades, y que facilite la búsqueda y/o generación de trabajo.

\section{LA FORMACIÓN PARA EL TRABAJO, EL APRENDIZAJE EN EL TRABAJO Y LOS PROGRAMAS CON PRÁCTICAS EN EMPRESAS A TRAVÉS DE LOS AÑOS}

La "formación para el trabajo" constituye un concepto amplio, complejo y dinámico, que refiere a las habilidades, conocimientos y saberes socialmente necesarios, válidos y relevantes $^{5}$, que facilitan a los sujetos elegir, acceder, y/o permanecer en un trabajo, que mejoran su desempeño en los ámbitos laborales y su desarrollo como ciudadano (Gallart, 2008; Bowman, 2015). Asimismo, refiere a todas las instancias formales y no formales que ofrecen esos conocimientos y esas herramientas para desempeñarse en el mundo del trabajo, que no se agota en lo otorgado por el sistema escolar, sino que va más allá de la escuela (Riquelme, 2010), e incluye a variados actores, contextos, modalidades y estrategias, del ámbito público y del privado. ${ }^{6}$

La experiencia europea muestra que no existe una única respuesta al interrogante sobre si la formación de los jóvenes debe proveerse en las escuelas, en centros de formación o en

\footnotetext{
${ }^{4}$ La "empleabilidad", término presente en programas y propuestas desde '90, tuvo ciertas transformaciones/adaptaciones en el tiempo. Es entendida como un nexo tanto conceptual como práctico del trabajo decente, y abarca las habilidades, conocimientos, aptitudes y actitudes que aumentan la capacidad de los trabajadores para mantenerse en el mercado laboral o acceder a él.

${ }^{5}$ Construido en relación con un tiempo y lugar, donde el cambio tecnológico, las transformaciones del mundo laboral y las reformas en las políticas, son dimensiones que inciden sobre la orientación y el contenido de la formación (De Ibarrola, 2004). ${ }^{6}$ De Ibarrola (2004) distingue diferentes ámbitos desde dónde se ofrecen oportunidades de formación para el trabajo y destaca tres en particular: escolar (instituciones de nivel medio y superior), no escolar (espacios sociales vinculados a las organizaciones de la sociedad civil), y laboral (en los espacios de trabajo, ya sea a través de procesos informales o de capacitaciones formales programadas).
} 
las empresas (Jacinto, 1997), las investigaciones al respecto suelen señalar que cuanto más próxima es la capacitación a la situación real de trabajo, más posibilidades hay para la empleabilidad posterior del joven (Freyssinet, citado por Jacinto, 1997).

Las nociones propuestas en la bibliografía internacional coinciden en señalar que el "Aprendizaje Basado en el Trabajo" (ABT)7 integra esferas: es aprender para trabajar, aprender en el trabajo y aprender a través del trabajo (BIBB, 2016), por ende está fundado en la práctica y la experiencia; implica la participación de la persona (aprendiz) en la realización de tareas laborales; y está situado (contextualizado en el lugar de trabajo y con las personas que forman parte del proceso).

En el caso de Argentina, se vienen promoviendo algunas políticas que incluyen componentes de ABT (Jacinto y Pozzer, 2019). Estos formatos de aprendizaje se concibieron como complementos útiles de la formación teórica y práctica en el ámbito del sistema educativo, dado que introducen a los estudiantes a las rutinas y rituales de un lugar de trabajo real y a los códigos profesionales, valores y normas de una ocupación y un entorno de empleo (Jacinto y Pozzer, 2019).

No obstante, el desarrollo y la implementación de un sistema que brinde a los jóvenes la oportunidad de aprender en un lugar de trabajo real se enfrentan a muchos desafíos. Gallart (2001) señalaba que el mayor reto de estos dispositivos reside en cómo desarrollar y consolidar un sistema, y cómo crear una cultura que valorice este componente e involucre a las partes interesadas en diferentes niveles de implementación (empresas, sindicatos, estado).

Estas discusiones se centraron fundamentalmente en torno al ámbito educativo formal, pero este formato comienza a ser utilizado también como propuesta de intervención en programas de empleo. A continuación presentaremos una breve descripción de los diferentes programas implementados que involucraron el desarrollo de prácticas en espacios "reales" de trabajo en empresas, como así también algunos resultados alcanzados.

\section{a) El "Proyecto Joven"}

Como señalamos, en los años `9o surgió un tipo de modelo de prácticas laborales en el marco de las políticas activas de empleo gestionadas desde el MTESS. Como resultado de las altas tasas de desempleo juvenil ${ }^{8}$, el gobierno nacional tomó varias iniciativas para intentar reducir la desocupación bajo el principio de elevar la "empleabilidad" y las "competencias" de una franja poblacional que se suponía tenía escasas calificaciones requeridas y valoradas para el mercado de trabajo, ya sea por no haber concluido la educación básica obligatoria o porque sus conocimientos no eran adecuados para ocupar un puesto calificado.

\footnotetext{
${ }^{7}$ Puede ser tanto formal como informal, según el grado de institucionalidad y sistematización del proceso, se realiza tanto en escuelas como en empresas, y contará o no -en uno u otro sistema- con planes de estudios estructurados; instructores certificados; calificaciones profesionales reconocidas; realizado o no de forma complementaria entre el aula institucional y el lugar de trabajo. ${ }^{8}$ En 1994 la tasa de desempleo PEA en el total de aglomerados relevados por la EPH-INDEC era del 11\%, mientras que para la población joven (menores de 25 años) ascendía al 22\%, creciendo en años siguientes.
} 
Siguiendo la experiencia del "Programa Chile Joven", y con financiamiento del Banco Interamericano de Desarrollo (BID), el Tesoro Nacional, y con apoyo institucional del Programa de las Naciones Unidas para el Desarrollo (PNUD), el Proyecto Joven (1993-200o) constituyó la inversión más importante de la década orientada a la formación laboral de jóvenes desempleados con bajos niveles educativos. ${ }^{9}$

Sus objetivos principales eran satisfacer dos tipos de demandas, por un lado, la "formación de mano de obra", y de esta manera apoyar la "reconversión" de los sectores productivos del país; por otro lado, estaba orientado a lograr un incremento de las posibilidades de inserción laboral y social de jóvenes considerados socialmente vulnerables (Mitnik, 1997). Las líneas de intervención se centraron en la capacitación técnica, pasantías y apoyo a la inserción laboral.

Para su ejecución, el modelo consistió en la externalización de la oferta de cursos a través de instituciones de capacitación privadas (ICAPs), seleccionadas mediante un proceso de licitación..$^{10}$ Se trataba de cursos cortos de capacitación en oficios, gratuitos, de naturaleza eminentemente práctica, cuyos contenidos eran diseñados por dichas instituciones en respuesta a las demandas de mano de obra que planteaban las empresas de su zona de influencia. Finalizada la instancia de capacitación -que tenía una duración máxima de 3 meses-, los participantes debían realizar pasantías en empresas -también se extendían por 3 meses-. Supervisados por las ICAPs, los beneficiarios se desempeñarían en un puesto de trabajo acorde con la ocupación para la que habían sido capacitados (Barbetti, 2010; Devia, 2003; Mitnik, 1997).

Participaron más de 115.000 jóvenes ${ }^{11}$ y 25.000 empresas $^{12}$ en todo el país brindando espacios para las prácticas. Si bien algunas evaluaciones (Devia, 2003; Jacinto, 1997; Mitnik, 1997) marcaron cuestiones positivas, se detectaron debilidades en la instancia de las pasantías vinculadas: a) la gestión de las ICAPs y las demoras en las firmas de los convenios, que desalentaban a las empresas a participar; b) la inexistencia de estudios previos sobre las necesidades de capacitación; c) el inadecuado seguimiento y cumplimiento de la función tutorial.

Por parte de las empresas, Sergio Devia (2003) señala que los puntos de atracción para participar se vincularon a la utilización del beneficio como un mecanismo para el reclutamiento y selección de personal de bajo costo, y en algunos casos como un aporte a su productividad media durante la ejecución de las prácticas por contar con un nuevo "colaborador" en la realización de las tareas de baja calificación. No obstante, el autor señala algunos resultados de las pasantías desde la mirada de las empresas, quienes consideraron que la práctica en el

\footnotetext{
${ }^{9}$ Requisitos para ser beneficiario: edad mínima de 16 años y preferentemente no mayor de 35 años; nivel educativo no mayor a secundario completo; desocupados o subocupados; pertenecer a hogares de nivel socioeconómico bajo; no asistir en horario diurno a una institución educativa de nivel primario o medio. Perfil de los beneficiarios participantes: $74 \%$ de 16 a 24 años de edad; $92 \%$ hasta secundario incompleto; $83 \%$ desocupados.

${ }^{10}$ Otorgó al mercado un rol preponderante en la asignación de recursos e identificación de beneficiarios; y redefinió el rol del Estado como contratante, supervisor y financiador de la capacitación dirigida al grupo focalizado, delegando el rol de diseñador y ejecutor directo de la capacitación.

${ }^{11}$ En Chaco fueron cubiertos 1471 jóvenes (80\% varones y 20\% mujeres) y 1924 en Corrientes (69\% varones y 31 mujeres).

${ }^{12}$ El $28 \%$ fueron microempresas (1-5 personas), el $44.2 \%$ son pequeñas (6-25 personas), el $19 \%$ son medianas (26-100 personas) y el $8,8 \%$ son grandes (más de 100 personas). Con respecto al sector económico: el 61,3\% pertenecen al sector servicios; el 27,7\% a industria; el 7,8\% a construcciones, agua, energía, gas.
} 
lugar de trabajo incrementó las competencias técnico operativas de los pasantes, dado que consolidaban los conocimientos adquiridos durante la primera etapa. En tanto los tutores de las pasantías afirmaron que los jóvenes adquirieron hábitos laborales -disciplina, puntualidad, presentación personal, responsabilidad-; y la participación en este programa como "acreditación" de experiencia previa.

En términos de impacto sobre las trayectorias laborales de los jóvenes, Moura Castro (1997) señalaba que pasados tres años de implementación del Programa, al menos el 56,3\% de los hombres y el 64,5\% de las mujeres no eran contratados por las empresas donde habían realizado su práctica laboral. El análisis de Devia (2003) muestra que esto se mantuvo a lo largo de su ejecución dado que fue muy bajo el impacto en la inserción laboral de los beneficiarios en puestos de trabajo en la especialidad del curso realizado, obtenida al año de finalizado el curso.

Desde la perspectiva de los jóvenes, su participación en la capacitación daba la posibilidad de adquirir una herramienta que facilite la inserción laboral. En esta línea, Jacinto (1997) señala que, además de esa motivación general, han identificado tres aspectos especialmente valorados: el carácter práctico de la formación, la beca percibida y la oportunidad de realizar alguna pasantía en un lugar de trabajo. Particularmente esta última asumió un valor de una instancia de capacitación en el trabajo como también de proveedora de una oportunidad de acceso al mercado en un circuito laboral que el joven, por su propio capital social, no hubiera logrado (Jacinto, 1997). Las críticas sobre esta instancia se centraron en el desajuste existente entre la práctica y lo estudiado y en la falta de apoyo de los tutores.

De modo general se puede decir que, el impacto positivo dependía estrechamente de las condiciones del mercado laboral y del tamaño y diseño individual del programa. Al respecto, debemos recordar que la propuesta fue ejecutada durante un período de aumento masivo en las cifras de desempleo. En consecuencia, sus efectos sobre la integración de estos jóvenes en el mercado laboral fueron muy bajos. Finalizó hacia fines del 2000.

\section{b) El "Programa Jóvenes con Futuro" (PJF)}

Recién en 2007, se puso en marcha una "experiencia de formación y aprendizaje basada en el trabajo", tipificada como una intervención pública-privada ${ }^{13}$ de cobertura y escala acotada a casi 2000 jóvenes del Área Metropolitana de Buenos Aires y de las ciudades de Córdoba y Mendoza (Lépore, 2015).

Promovido por el MTESS y apoyado en acciones de "responsabilidad social" de 26 grandes empresas y 2 cámaras empresariales, se enfocó en jóvenes con dificultades para acceder a un empleo formal. ${ }^{14} \mathrm{Un}$ aspecto interesante y distintivo de este programa es que las empresas

\footnotetext{
${ }^{13}$ Desarrollado y financiado en el marco de la Red de RSE y Trabajo Decente impulsada por el MTESS, fue establecido por Convenio 164/06 entre el Ministerio y las empresas del sector privado.

${ }^{14}$ Dirigido a jóvenes de 18 a 24 años, de sectores vulnerables, que se encuentran en dificultades de acceso a un empleo por no haber completado sus estudios secundarios y por no contar con una formación profesional acreditada o una experiencia laboral significativa.
} 
reconocen entre los principios organizadores su responsabilidad social en la generación de oportunidades de capacitación y práctica laboral para aquellos jóvenes que presentan mayores dificultades de inserción.

La propuesta vinculaba actividades de formación profesional en el aula (que podían darse en convenio con alguna institución educativa) con prácticas en puestos de trabajo dentro de las empresas convocantes, buscando el desarrollo de las habilidades básicas y las competencias técnicas y laborales requeridas por cada ocupación. Además incluía acciones de guía y acompañamiento tendientes a lograr la finalización de los estudios formales y la alfabetización digital. La propuesta podía tener una duración de entre 3 y 10 meses dependiendo del contenido de cada proyecto formativo, los cuales estaban en relación con los perfiles ocupacionales para los cuales se capacitaba a los jóvenes.

El acompañamiento del joven era realizado por un tutor de la empresa. Un informe del programa (MTESS, 2010) resalta la presencia y trabajo efectivo por parte del tutor con el joven, a través del acompañamiento y desarrollo de evaluaciones continuas. Esto aparece destacado como un ámbito de aprendizaje surgido del seguimiento personalizado.

La evaluación de resultados del PJF (OIT y MTESS, 2010) sobre los actores involucrados muestran varios efectos positivos. Por el lado de las empresas dan cuenta de cambios internos sobre la capacidad adquirida para movilizar recursos personales y poner en marcha trabajos conjuntos entre distintas gerencias o departamentos, como así también sobre las políticas de selección de personal, que lograron remover ciertos prejuicios en las tareas de reclutamiento, especialmente relacionados con la procedencia social de los trabajadores. La articulación con el sector público sirvió tanto para iniciar un relacionamiento más estrecho con éste, como para consolidar las políticas empresariales de RSE mediante una asociación público-privada. En varios casos señalan que su implementación continuaría aún en caso de no contar con el apoyo del sector público, incorporando este sistema de reclutamiento y formación como parte de su política de Recursos Humanos.

En tanto, por parte de los jóvenes, se alcanzaron buenos resultados en términos de acceso al empleo: 59 \% de los participantes se encontraba ocupado en un empleo registrado al terminarlo (49\% por las mismas empresas donde realizaron la práctica) y en igual proporción retomaron los estudios formales (OIT-CINTERFOR). Al momento de la finalización del ciclo, las empresas capacitadoras se comprometen a difundir los perfiles ocupacionales de los jóvenes no incorporados en su planta entre aquellas que integran su cadena de proveedores, distribuidores y subcontratistas. Adicionalmente, brindaron apoyo y orientación laboral a los jóvenes "egresados" de las prácticas, para facilitar la búsqueda de empleo posformación.

De acuerdo con los datos recabados en una encuesta realizada desde el PJF a los jóvenes participantes, para muchos constituyó su primera experiencia de inmersión en el mundo del trabajo, aunque se señala que no se perdía de vista el carácter formativo de la práctica. Asimis- 
mo, entre los aspectos positivos destacaban el desarrollo de habilidades "sociales" (fundamentalmente la posibilidad de aprender a trabajar en equipo); adquirir experiencia para conseguir trabajo; tener un ingreso fijo durante el período de la capacitación y de aprender un oficio o trabajo concreto (OIT y MTESS, 2010). Conjuntamente, enfatizaron en el aprendizaje de aspectos que hacen a la "cultura de un trabajo de calidad" (cumplimiento de horarios, relacionarse con las distintas jerarquías, etc., así como la formación en derechos laborales, salud y seguridad en el empleo). Las críticas se vincularon a problemas en la gestión administrativa del programa (cierta desorganización, demoras en el pago de asignación y disconformidad con el monto recibido).

Ahora bien, las condiciones extremadamente acotadas y supervisadas del programa, sumadas al compromiso particular de las empresas como parte organizadora, fueron claves para estos resultados y plantean el interrogante acerca de las posibilidades de ampliar la escala del modelo.

\section{c) Programa Jóvenes con Más y Mejor Trabajo (PJMMT)}

Desde 2008 (y continua activo) se lanzó un nuevo programa masivo en el marco de las políticas activas de empleo. Esta iniciativa, financiada por el Banco Mundial, e implementada por el MTEySS, se dirige a jóvenes de entre 18 y 24 años que estén desocupados y no hayan finalizado sus estudios secundarios. Se inserta en el Plan Integral de Empleo "Más y Mejor Trabajo" que marca la transición hacia políticas activas en base a una estrategia novedosa de articulación sectorial y territorial, de gestión descentralizada.

La propuesta programática corresponde a lo que se denomina "modelos integrados" (Dema et. al., 2015), dado que ofrece un conjunto amplio de acciones orientadas a servir de apoyo en la construcción e implementación de un proyecto formativo-ocupacional. Así vemos que promueve que los participantes puedan identificar el perfil profesional en el cual deseen desempeñarse, finalizar su escolaridad obligatoria, realizar experiencias de formación y/o prácticas calificantes en ambientes de trabajo, iniciar una actividad productiva de manera independiente o insertarse en un empleo (Art. $1^{\circ}$ Resolución 497/o8).

La implementación de las prestaciones a nivel local se enmarca en la conformación de un diálogo social ampliado, basado en una gestión conjunta entre los gobiernos provinciales y la red de Oficinas de Empleo (OE), en articulación con instituciones educativas y de formación profesional, organizaciones empresariales, sindicales y de la sociedad civil. En este marco, un aspecto novedoso del programa es que apunta a una mayor institucionalidad -a diferencia de las medidas ad hoc que habían caracterizado al Proyecto Joven- y al fortalecimiento de la coordinación local, promoviendo articulaciones entre distintos actores que configuran la relación educación-formación-trabajo (Roberti, 2018).

Ahora bien, el contexto del mercado laboral de Chaco y Corrientes, ha sido caracterizado por el insuficiente desarrollo de sus estructuras sociales y productivas, presentando históri- 
camente índices de pobreza e indigencia superiores a la media nacional. Investigaciones sobre la región (Golovanevsky, 2012; Barbetti et al. 2015; INDEC, 2018) dan cuenta de que los jóvenes son quienes conforman uno de los grupos con mayor grado de vulnerabilidad, dado que muestran altos índices de inactividad, menor permanencia y estabilidad en las ocupaciones, realizando tareas no calificadas, con escasas posibilidades de desarrollo, y condiciones de contratación más precarias. Además, algunas características del contexto empresarial local señalan una fuerte concentración de microempresas, vinculadas a los sectores de servicios y comercio, y existe una importante cantidad de trabajadores asalariados no registrados dentro del sector privado (INDEC, 2018).

A lo largo de los últimos años, se ha observado la continuidad en la gestión de esta línea programática de "Entrenamientos para el Trabajo" (EPT) en los servicios de empleo de las ciudades de Resistencia y de Corrientes, en articulación con empresas de la zona.

Tomando como base la propuesta del PJF, la práctica se desarrolla en ambientes laborales reales, pueden durar hasta 6 meses (48ohs.) y realizarse en organizaciones del sector público, sociales o en empresas privadas. ${ }^{15}$ Pueden contener componentes de formación teórica (no es obligatorio) relacionada a la ocupación y formación práctica en el puesto de trabajo. En el establecimiento donde desarrollan la práctica, los jóvenes deben contar con la guía y seguimiento de un tutor designado por la empresa o institución organizadora.

La intermediación con empresas es realizada por las oficinas de empleo locales. Algunos datos muestran que, a nivel nacional, más de 204.952 jóvenes desarrollaron estas acciones, aunque solo representan a poco más del 10\% de los participantes del Programa Jóvenes (MTESS, 2014). El 77\% de ellos no había finalizado la escuela secundaria, y el 90\% contaba con alguna experiencia laboral, aunque casi la totalidad era informal (Lépore, 2015).

Los últimos datos de la Secretaría de Empleo (MTESS) a los que hemos tenido acceso en 2015, mostraban que la prestación EPT tenía una gran dispersión entre los servicios de empleo en el país: de un total de 297 OE, sólo 4 de ellas tuvieron más de 1000 jóvenes desarrollándolos Rosario (3394), La Matanza (2896), Ciudad de Buenos Aires (2041) y Resistencia (1281).

Siguiendo el marco y la lógica de implementación del programa -centrada en el nivel local-, a partir de algunos resultados de investigación propios, en los servicios de empleo de las ciudades de Resistencia y Corrientes, podemos señalar algunas cuestiones de su gestión desde la perspectiva de diferentes participantes (agentes estatales, empresas y jóvenes).

En el caso de la Resistencia, el EPT es una línea que se viene implementando desde el año 2010, y participaron más de 400 empresas (datos de julio de 2015 de la Agencia Provincial de Empleo). La situación en la ciudad de Corrientes fue diferente, empezó a gestionarse en períodos de tiempo más recientes. Algunos datos muestran que entre los años 2015 y 2016, fueron 154 empresas las que suscribieron acuerdos de EPT y pudieron desarrollarlos 393 jóvenes (Informes de gestión de la OME Corrientes, 2015 y 2016). 
Un actor clave en este dispositivo es el servicio local de empleo. Desde ellos se visualiza el bajo porcentaje de jóvenes que realizan EPT, lo cual marca la complejidad de alcanzar acuerdos con empresas para la apertura de espacios de trabajo para realizar prácticas. En un estudio previo (Pozzer, 2016) han descrito cuestiones vinculadas a la limitada cultura empresarial de desarrollar este tipo de experiencias y la desconfianza por parte del empresariado en relacionarse con el Estado. Asimismo, identifican las dificultades dentro del servicio de empleo para definir los perfiles de puestos de prácticas y el de los jóvenes a derivar como parte de los procesos de orientación, y para ajustar las expectativas de los empresarios solicitantes. Otros puntos críticos se vinculan con el seguimiento durante la práctica, y la tutoría que se circunscribe a la instancia de intermediación, cuando inicia el EPT queda totalmente a cargo de la empresa (Jacinto y Pozzer, 2019).

Desde la mirada de las empresas, las prácticas se constituyen en un modo de gestión de personal mediante el que se cuenta con un "período de prueba" en el cual identificar perfiles jóvenes y evaluar desempeños de potenciales personas a contratar. Para varios casos significó contar con mano de obra que en forma temporal permite dar una respuesta rápida y económica ante una expansión de la demanda. ${ }^{16}$ También aparecen discursos de la importancia de participar en este tipo de propuestas como parte de Responsabilidad Social Empresaria.

Mayoritariamente, los EPT se realizan en puestos con tareas operativas de baja complejidad, en el marco de micro-pequeños y medianos establecimientos. En general, éstos muestran bajos niveles de desarrollo organizacional, lo cual dificulta crear entornos calificantes. La posibilidad de incluir acciones de tutoría organizadas y sistematizadas, donde se analicen los aprendizajes de cada participante es limitada.

A la vez, hemos identificado ciertos casos de empresas donde cuentan con áreas de gestión de personal, en las cuales el nivel de organización y planificación de los EPT aparece desarrollado (definición y selección de perfiles, actividades de capacitación, esquemas de rotación predefinidos, evaluación de desempeño, entre otros).

Independientemente del tipo de empresa que se trate, la actividad de tutoría "recae" en el responsable/autoridad jerárquica del área en que se realizará el EPT, se observa que no hay características/preparación previa para asumir ese rol. El aprendizaje del entrenando se desarrolla en el puesto de trabajo, se privilegia la práctica, el tutor es quien oficia como transmisor de los saberes necesarios para realizar las actividades.

Desde la perspectiva de los jóvenes participantes, enfatizan la valoración del entrenamiento en la adquisición de competencias transversales (responsabilidad, puntualidad, cumplimiento de horarios, compromiso con la tarea realizada) por sobre las específicas del puesto, y en la utilidad para mostrar una experiencia y referencias en la búsqueda de un nuevo empleo. Asimismo, aparece la motivación de participar como un medio para el desarrollo de habilidades útiles para generar otro tipo de proyecto propio que no se vincula al empleo. Es importante

\footnotetext{
${ }^{16}$ Los beneficiarios reciben una asignación estímulo no remunerativa mensual y un complemento en concepto de compensación por gastos de traslado financiado conjuntamente por el MTESS y la empresa participante. La empresa toma a su cargo el pago de un Plan Médico Obligatorio, de un Seguro de Riesgos del Trabajo, y de los seguros de responsabilidad civil que corresponden a la normativa vigente.
} 
señalar que para varios jóvenes (particularmente provenientes de contextos más vulnerables) fue valorado por la posibilidad de contar con una fuente de ingresos y por acceder a un espacio de integración social y contención del que carecían (Pozzer, 2015; MTESS 2014).

\section{A MODO DE CIERRE}

De manera general podemos señalar cierta continuidad en las acciones sobre la problemática de la inserción de los jóvenes, aunque partiendo de diferentes "causalidades", las estrategias recaen sobre la individualización y centran la acción sobre la empleabilidad. En el marco del paradigma de la activación, se buscó en cierto modo proveer al individuo de las herramientas adecuadas para facilitar la gestión individual del riesgo, más que protegerlo frente a éste (Roberti, 2018). Si bien, a nivel discursivo y programático, se advierten cambios en la complejidad para abordar las transiciones al trabajo, los programas implementados ponen escaso énfasis en las condiciones de generación de empleo y de trabajo ofrecidas. Así hay quienes sostienen que las transformaciones no han sido tan profundas, señalando continuidades en torno al carácter asistencial y compensatorio de las políticas a lo largo de estos años.

Particularmente, los componentes que plantean la articulación con empresas para realizar prácticas, continúan generando resultados similares. A lo largo de este trabajo, se ha descrito cómo en los 90 se articuló la pasantía con periodo de capacitación en el aula para el desempeño de una ocupación específica, "atendiendo" al problema de la inserción generado por la carencia de "capital humano". Avanzados los años 2000, el entrenamiento buscó generar competencias generales que no se limitaran a un puesto/ocupación particular, y que se desarrollaran en la misma práctica y no en las aulas.

Asimismo hemos visto que el formato ha sido gestionado de diferentes maneras: a través de instituciones privadas o mediante la intermediación de los servicios estatales de empleo. Sin embargo, y teniendo siempre presente que las condiciones macro estructurales y contextos de implementación coadyuvan a los resultados de toda política pública, las pasantías-prácticas como dispositivos activos de empleo han mostrado que los obstáculos y dificultades para alcanzar resultados con alto impacto se reiteran a pesar de formas diferenciadas de implementación.

Específicamente, continúan despertando un escaso interés de la parte empleadora; como así también las lógicas de gestión y ejecución: escaso seguimiento y acompañamiento de los jóvenes, limitado potencial formativo y bajo nivel de incorporación laboral, etc. Exceptuando el programa de escala acotada, propuesto y gestionado desde la "responsabilidad social" de las empresas, parecen no generar mayor compromiso en este actor fundamental de las relaciones laborales. 
En cierto punto esto lleva a plantearnos ċlas empresas no tienen interés en la población con la que trabajan los programas, ya que podrían cubrir sus necesidades con jóvenes que demuestran "mejores" antecedentes? En relación con lo anterior, si existe demanda por parte de las empresas que se vincule a los perfiles propuestos en estos programas ciel "mercado" tiende a asignar en esos puestos a los beneficiarios?, o en el peor de los escenarios ise utilizan como medio de "contrato" a desocupados con mayores calificaciones que estén dispuestos a aceptar condiciones precarias de vinculación laboral? No podemos pasar por alto el contexto en que se gestionan estos programas, en la región NEA se complejiza aún más considerando que entre los trabajadores asalariados casi un 40\% están en condiciones de informalidad (Salvia et. al., 2018).

Desde las primeras experiencias, se ha venido advirtiendo que los sistemas de práctica en espacios de trabajo permiten incorporar personal financiado por el Estado, pero con un subsidio que se orienta a reforzar la demanda de trabajo del empresariado, antes que a la protección social de los jóvenes desempleados. Lo Vuolo (2001) planteaba si este tipo de intervenciones no haría posible un "efecto sustitución" entre empleos genuinos y las prácticas laborales temporales promovidas de este modo. Estas reflexiones no niegan la potencialidad de estos dispositivos, sino buscan poner en tensión ciertas prácticas del actor empresarial y el modo en que se gestionan.

Asimismo, a nivel micro-individual se ha mostrado que pueden contribuir a mediar y mejorar la posición de los jóvenes en sus estrategias de acercamiento y acceso al mercado de trabajo, sobre todo aquellos con menores capitales sociales y educativos.

$\mathrm{Al}$ respecto, sin lugar a dudas el PJMMT, ha sido un gran avance hacia la articulación de diferentes tipos de acciones que contribuyan a mediar en la inserción laboral del grupo juvenil, pero se presentan desafíos en cuanto a la implementación y gestión del componente específico. A corto y mediano plazo los esfuerzos parecen apuntar a la generación de interés y la articulación con los actores socio-productivos del mundo del trabajo formal, como así también a reforzar las instancias de tutorías y monitoreo de los EPT, a fin de evitar situaciones donde se desvirtúen sus objetivos, considerando las condiciones de trabajo en la región.

\section{REFERENCIAS BIBLIOGRÁFICAS}

Barbetti, P. (2010) "Estrategias de inclusión socio-laboral juvenil. Acerca del papel del Estado, las Empresas y la Sociedad Civil en los diseños normativos de las políticas públicas". Ponencia presentada en las Jornadas Nacionales sobre Estudios Regionales y Mercado de Trabajo, Facultad de Humanidades y Ciencias de la Educación de la Universidad Nacional del La Plata, junio de 2010. 
Barbetti P., Pozzer J. y Sobol B. (2015) Situación laboral de los Jóvenes en el Gran Resistencia y Corrientes, Argentina, en el período 2010-2013. Disponible en: Revista de la Facultad de Ciencias Económicas UNNE, Argentina. $\mathrm{N}^{\circ} 13$. Disponible en: http://revistas.unne.edu.ar/ index.php/rfce/article/view/390

BIBB (2016) Final Report of Mapping the research about workbased learning from a global TVET perspective - A literature review. Bonn: BIBB.

Bowman, M. (2015) “Formación para el trabajo y educación básica en jóvenes de baja escolaridad”. Tesis de Doctorado en Educación de la Universidad Nacional de Córdoba.

De Ibarrola, M. (2004) "Paradojas recientes de la educación frente al trabajo y la inserción social”. Instituto Internacional de Planeación de la Educación, RedETIS, IDES, Buenos Aires.

Dema, G.; Díaz, J. y Chacaltana, J. (2015) ¿Qué sabemos sobre los programas y políticas de Primer Empleo en América Latina? Lima: OIT.

Devia, S. (2003) ¿Éxito o fracaso de las políticas públicas de capacitación laboral a jóvenes? Evaluación del programa testigo: "Proyecto Joven" de Argentina (1993-200o). Tesis de Magister de la Universidad de Buenos Aires en Administración Pública.

Gallart, M. A. (2008) Competencias, productividad y crecimiento del empleo: el caso de América Latina. Montevideo: OIT/Cinterfor.

Gallart, M. A. (2001) El desafío de la formación para el trabajo de los jóvenes en situación de pobreza: el caso argentino. En Gallart, M. A Formación, pobreza y exclusión. CINTEFOR. Págs. 241-311. Montevideo.

Golovanevsky, L. (2012) Inserción laboral de los jóvenes en la pos convertibilidad: una visión regional. Ponencia presentada en II Jornadas Nacionales sobre Estudios Regionales y Mercados de Trabajo. Santa Fe, UNL.

INDEC (2018) Informe de diagnostico laboral-Provincia de Corrientes. Estudios y Estadísticas del Mercado de Trabajo del Ministerio de Producción y Trabajo.

Jacinto, C. (2015) "Nuevas lógicas en la formación profesional en Argentina. Redefiniendo lo educativo, lo laboral y lo social", Perfiles Educativos, vol. XXXVII, N $^{\circ} 148$. 


\begin{tabular}{c}
\hline JOSÉ ANTONIO POZZER \\
PRÁCTICAS EN LUGARES DE TRABAJO EN LOS PROGRAMAS DE EMPLEO JUVENIL: \\
Págs. $79-99$. DOI: $h t t p: / / d x$. doi.org/10.30972/rfce.0213729
\end{tabular}

Jacinto, C. (2010) Veinte años de políticas de formación para el empleo de jóvenes vulnerables en América Latina: persistencias y reformulaciones. En: Claudia Jacinto (Comp.) La construcción social de las trayectorias laborales de jóvenes. Políticas, instituciones, dispositivos y subjetividades. Buenos Aires: Teseo-IDES.

Jacinto, C. (2008) "Los dispositivos recientes de empleo juvenil: institucionalidades, articulaciones con la educación formal y socialización laboral”. En Revista de Trabajo, Año 4, №6. Buenos Aires: MTEySS.

Jacinto, C. (1997) Políticas públicas de capacitación laboral de jóvenes. Un análisis desde las expectativas y las estrategias de los actores. En Revista Estudios del Trabajo, No ${ }^{\circ}$. Buenos Aires: ASET.

Jacinto, C. y Millenaar, V. (2009) Enfoques de programas para la inclusión laboral de los jóvenes pobres: Lo institucional como soporte subjetivo. En Revista Última Década [en línea], 30, 67-92.

Jacinto, C. y Pozzer, J. (2019) Work-based learning as a concept under construction? Evidence from two different internship schemes in Argentina. In Bahl, Anke; Dietzen, Agnes (Eds.) Work-based Learning as a Pathway to Competence-based Education. UNEVOC Network Contribution. Bonn.

Lasida, J. (2004). Estrategias para acercar a los jóvenes al trabajo. Buenos Aires: redEtis.

Lépore, E. (2015). "Políticas de empleo e inserción laboral juvenil. Resultados e impactos de experiencias recientes en la Argentina". En VIII Congreso Latinoamericano de Estudios del Trabajo. Facultad de Ciencias Económicas de la Universidad de Buenos Aires.

Lo Vuolo, R. (2001) Alternativas: la economía como cuestión social. Buenos Aires: Altamira.

Madoery, O. (2011) Más y mejor trabajo para todos. Del programas jefes de hogar al programa jóvenes. Políticas activas de empleo, Argentina 2003-2010. Sistematización y análisis integrado. Programa CEA-OIT. Buenos Aires: OIT.

Mitnik, G. (1997) Proyecto Joven: la capacitación laboral como herramienta de equidad social. Boletín CINTERFOR N ${ }^{\circ}$ 139-140. Montenvideo: Cinterfor/OIT. 
Moura Castro, C. (2002) "Formación de jóvenes desempleados en América Latina”, en Castro, C. de Moura; Schaack, K; Tippelt, R. (Eds.) Formación profesional en el cambio de siglo. Montevideo: Cinterfor/OIT.

Neffa, Julio C. (coord.) (2012). Empleo, desempleo y políticas de empleo: De las políticas pasivas a las políticas activas de empleo: análisis comparativo (1989-2011). Buenos Aires: CEILPIETTE.

OIT (2016) Soluciones eficaces: Políticas activas del mercado de trabajo en América Latina y el Caribe / Oficina Internacional del Trabajo. Ginebra: OIT.

OIT (2012) Competencias para el empleo. Orientaciones de política: Impulso a la empleabilidad de los jóvenes desfavorecidos. Ginebra: Departamento de Conocimientos Teóricos y Prácticos y Empleabilidad Sector del Empleo. (OIT - Competencias).

Partenio, F. (2015) Aprendizaje de calidad y preparación de los jóvenes para el trabajo en Argentina. Montevideo: OIT/Cinterfor.

Pérez, P. (2013) "Empleabilidad, motivación para trabajar y políticas de empleo para jóvenes en Argentina”. La Plata, FaHCE UNLP.

Pérez, P. y Brown, B. (2015) Políticas de Empleo para jóvenes: el programa Jóvenes con y más y mejor trabajo. En Pablo Pérez y Mariana Busso (coord.) Tiempos Contingentes: inserción laboral de los jóvenes en la Argentina Posneoliberal. Buenos Aires: Miño y Dávila.

Pozzer, J. (2016) Alcances y limitaciones de los Entrenamientos para el Trabajo como dispositivo de formación e inserción en el empleo. Un estudio de caso en Resistencia, Chaco. En Jacinto C. (coord.) Protección social y formación para el trabajo de jóvenes en la Argentina reciente. Buenos Aires: IDES.

Pozzer, J. (2015) Transiciones juveniles al mercado laboral. Dispositivos y subjetividades de jóvenes participantes de acciones de entrenamiento en el trabajo. Ponencia presentada en el $12^{\circ}$ Congreso ASET, Agosto de 2015, Facultad de Ciencias Económicas, Universidad de Buenos Aires.

Riquelme, G. y Herger, N. (2001) Acceso a la educación y formación para el trabajo: ¿Quiénes y qué tipos de cursos? Ponencia presentada en el $5{ }^{\circ}$ Congreso ASET, Agosto de 2001, Facultad de Ciencias Económicas, Universidad de Buenos Aires. 


\begin{tabular}{c}
\hline JOSÉ ANTONIO POZZER \\
PRA \\
PRÁCTICAS EN LUGARES DE TRABAJO EN LOS PROGRAMAS DE EMPLEO JUVENIL: \\
Págs. $79-99$. DOI: $h t t p: / / d x$. doi.org/10.30972/rfce.0213729
\end{tabular}

Roberti, M. (2018) Políticas de inclusión socio-laboral para jóvenes: Un análisis de las trayectorias de participantes de programas de empleo (Prog.R.Es.Ar y PJMMT) en el Conurbano Bonaerense. Tesis de Doctorado. Universidad Nacional de La Plata. Facultad de Humanidades y Ciencias de la Educación. En Memoria Académica. Disponible en: http://www.memoria.fahce.unlp.edu.ar/tesis/te.1515/te.1515.pdf

Salvia, A.; Fachal, N. y Robles, R. (2018) Estructura social del trabajo. En Piovani J. y Salvia A. (coord.) La Argentina en el siglo XXI. Buenos Aires: Siglo Veintiuno editores.

Salvia, A. (2013) "Juventudes, problemas de empleo y riesgos de exclusión social. El actual escenario de crisis mundial en la Argentina”. Berlin, Fundación Friedrich-Ebert-Stiftung.

Salvia, A. (comp.) (2008) Jóvenes promesas. Trabajo, educación y exclusión social de jóvenes pobres en la Argentina. Buenos Aires, Miño y Dávila.

Salvia, A. y Tuñón, I. (2003): "Los jóvenes trabajadores frente a la educación, el desempleo y el deterioro social en la Argentina”. Friedrich Ebert Stiftung Argentina. Buenos Aires.

\section{NORMATIVA E INFORMES:}

Informes de gestión y resultados de la Oficina Municipal de Empleo de la ciudad de Corrientes 2015 y 2016. Dirección General de Empleo, Subsecretaria de Desarrollo Económico de la Secretaria de Desarrollo Productivo y Economía Social de la Municipalidad de la Ciudad de Corrientes.

Ley Nacional de Empleo N$^{\circ} 24.013 / 91$ de la Republica Argentina.

MTESS (2014) Documento de trabajo "Programa Jóvenes con Más y Mejor Trabajo. Revisión de Entrenamientos para el Trabajo-Estudio de casos”. No publicado, febrero de 2014.

OIT y MTESS (2010) Programa Jóvenes con Futuro: resultados e impactos de una intervención público-privada. OIT Argentina y MTESS: Buenos Aires.

Resolución No 696/2006 MTEySS, Buenos Aires, 14/07/2006.

Resolución Nº 497/2008 MTEySS, Buenos Aires, 13/05/2008. 
Resolución Nº 708/2010 MTEySS, Buenos Aires, 14/07/2010.

Resolución Nº 905/2010 SE-MTEySS, Buenos Aires, 27/07/2010.

Resolución N 879/2011 SE-MTEySS, Buenos Aires, 26/05/2011.

Resolución No 1032/2014 SE-MTEySS, Buenos Aires, 13/5/2014.

\section{COMO CITAR ESTE ARTÍCULO:}

POZZER, José Antonio. Prácticas en lugares de trabajo en los programas de empleo juvenil: experiencias, cambios y continuidades en su implementación. Revista de la Facultad de Ciencias Económicas - UNNE, Argentina. Volumen 21 Núm. 2, julio-diciembre 2018, ISSN 1668-6365. Págs. 79 - 99. DOI: http://dx.doi.org/10.30972/rfce.0213729

\section{CURRICULUM VITAE}

\section{José Antonio Pozzer}

Licenciado en Relaciones Laborales, egresado de la Universidad Nacional del Nordeste. Doctorando en el Doctorado en Ciencias Sociales de la Universidad Nacional de Entre Ríos. Becario interno doctoral del Consejo Nacional de Investigaciones Científicas y Tecnológicas (CONICET). Docente de la Facultad de Humanidades y de la Facultad de Ciencias Económicas, UNNE. Integrante de proyectos de investigación acreditados por la Secretaría General de Ciencia y Técnica (UNNE), desde 2010 hasta la actualidad. Integrante del Programa de Investigaciones sobre Juventud, Educación y Trabajo (PREJET) del Instituto de Desarrollo Económico y Social (IDES) y Centro de Investigaciones Sociales (CIS) CONICET Buenos Aires. Docente Investigador Categoría IV. Temas de investigación: formación para el trabajo; políticas públicas de empleo; trayectorias socioeducativas y laborales de jóvenes.

japozzer@yahoo.com.ar 